\title{
Abstraction of View Elements from Software Requirement Specifications (SRS)
}

\author{
Syed Naimatullah Hussain \\ Department of Computer Science, \\ Taif University \\ Taif, Saudi Arabia
}

\begin{abstract}
The software project development normally starts with customer's requirements. The customers will collect the requirements from the end users of the organization, and prepares a document called the SRS. Unified modeling language (UML) designed to provide a standard way to visualize the design of an information system. Apart from other internal lacunae of (UML) itself, the main reasons for low success rate are due to the non-availability of a correct and complete methodology for abstraction of the view elements for the design of an information system. This paper abstracts the view elements from Software Requirement Specification (SRS) for any information System in the form of Classes, Object of the Classes, Actors its interfaces their characterizing attributes, and these abstractions are further refined using good database design principles and the use of data flow diagrams (DFDs).
\end{abstract}

\section{Keywords}

Classes, Attributes, Interfaces

\section{INTRODUCTION}

The software development project normally starts with SRS of the customers $[1,2]$. The customers are in general, strategic management people of the organization who work in classical ambience. So the requirements of the expected system reflect their processing mindset. These requirements are influenced by either the data oriented approach or the procedure oriented approach as with the available information of the organization. Presently, since these are not natural ways of processing the information system, these will not serve the development process effectively. Now a day, people feel the object-oriented paradigm is more towards naturalness and will survive for long time. So it is required to transform the requirements into object-oriented paradigm (Classes, Object of Classes, Actors and its interfaces) and then proceed for the development. The main objective of this paper is to develop a system of software tools, that takes SRS and then transform it into Actors, Classes and Objects and its attributes.. There may also need to limit our ambition, as some of the sub processes may not be automated due to English as Natural Language. In such case, there is a need to provide guidelines for each of these sub processes to minimize the human dependency. the aim to develop a sequence of semi-automated software tools with embedded guidelines for inevitable subtasks at some stages then, the set of guidelines may give the scope to develop software agents to take up the role of semi automated processes.

Few researchers[3] have suggested some techniques for identification of object class hierarchies in procedural object oriented, but in our methodology we are using a perfect balance of procedural as well data oriented paradigm i.e nothing but object oriented paradigm which more towards naturalness for certain stages of the design of object classes. $[4,9]$ in this authors are abstracting only object class and its attributes based on parsed use case description not all the design components from the SRS. Author's $[8,10]$ this paper helps in the designing only the class and subclass structures but fails to identify the object class structure i.e. object class name, attributes, actors and its interfaces. Although, these give good guidelines to the design, the authors could not derive any concrete procedure and/or guidelines to the design in its totality. To develop a methodology that identifies Classes and Objects of the Classes, Actors and its attributes, , their characterizing attributes. This semi automatic methodology comprises of a sequence of steps like feasibility analysis, for object structure identification, resolution of synonyms \& homonyms issues, regrouping of attributes of entities \& functionalities through the design of data flow diagrams and elimination of imbalance data \& procedure selection along with authentication of correctness \& completeness of the abstractions at each stage. There is manual intervention at few stages is necessitated because of the need for human intelligence in these steps. Even for these manual intervention steps, attempt is made to provide clearcut guidelines to streamline the design process.

\section{METHODOLOGY}

The algorithm presumes that, based on SRS from customer's this SRS is already available with the developer. Who can seek needy information from client's team of users (CTU)? This paper addresses a sequence of semiautomatic methodology.

\subsection{Requirement Gathering}

As per the SRS, the detailed requirement is gathered from CTU. The input of any information system is SRS, output is Classes, Objects of the Classes, Actors and its attributes, If CTU is very large, and then similar information is collected through the response to the questionnaire from the client.

\subsection{Authentication of correctness and completeness of the process}

Now we have two sets of entities, attributes, interrelationships, business rules, work processes and business process. The developer need to establish the one-to-one and on-to correspondence separately between each pair of items of two sets.

\subsection{Resolve Synonyms/homonyms Issue}

As English is a Natural Language, in a multi-user system, though each user assigns meaningful names to attributes, the semantic flexibility in the use of English words leads synonymous words for the same attribute. The set of synonymous words of the same meaning forms a synonymy and each such synonymy is replaced by a generic name. Similarly, the use of context specific word leads to 
homonyms. The presence of each such homonym in attributes/entities/actors should be replaced by different names.

\subsection{Model the Business Process Through To Measure the Paradigms Imbalance}

- Entities, which are modifiable within the system

- Entities which are non modifiable within the systems form actors.

- The identified functionalities form processes

- The intersection of input/output attributes of a functionality with each entity attributes forms input/output dataflow from/to the concerned entity /actor to/form the process

This logical DFD (Fig-2.2) reveals the degree of imbalance in the paradigms opted while choosing structural and behavioral components. Figures 2.51 and 2.52 depict the possible reasons tilt respectively towards Procedure oriented and data oriented paradigms is more towards naturalness.

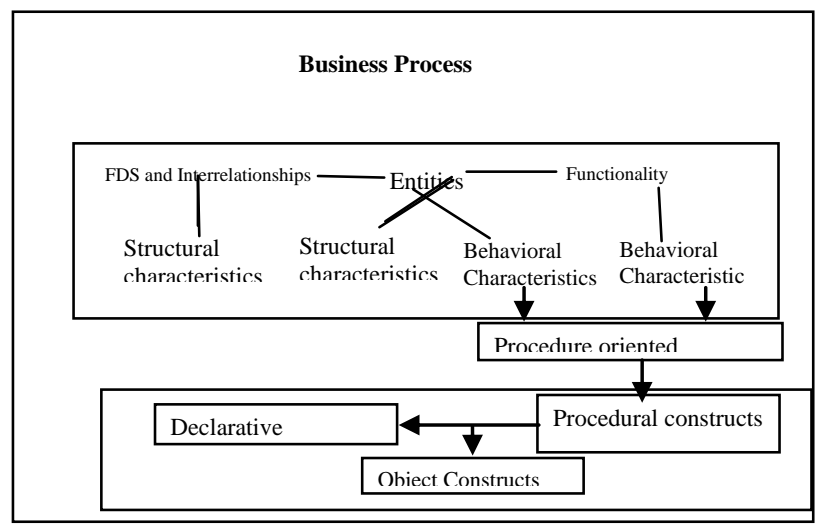

Fig. 1.1 Procedure Oriented

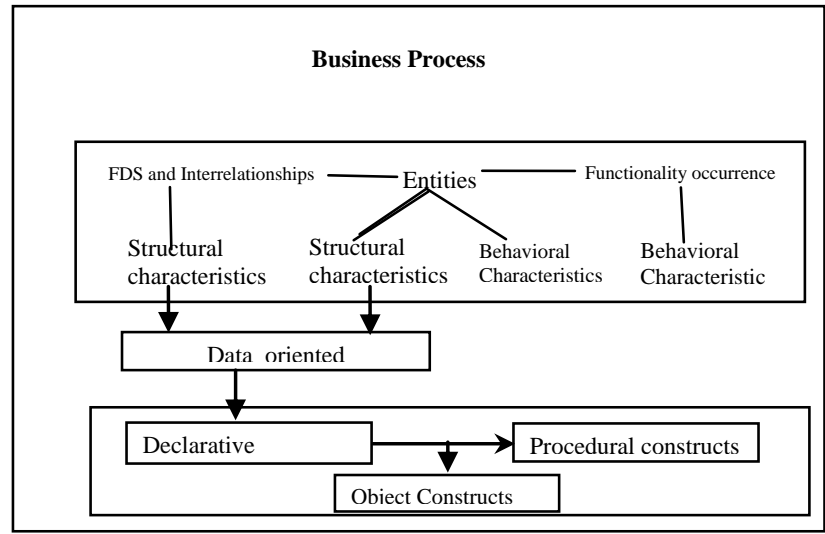

Fig.1.2 Data Oriented

\subsection{Eliminate Superfluity and Redundancy in Attributes/Entities Presence}

Study each attributes of each entity/actor in isolation with other entities/attributes for absolute necessity of their presence in the information system. This can be identified by the participation of the attribute in any of the functionalities. Discard the attributes that are not participating in the functionalities. If an attribute or group of attributes is present in two or more entities, form a separate entity with each such group. This participation can be tested through the establishment of one-to-one and on-to correspondence between attributes referenced in data flows of logical DFD (Fig 2.0)

\subsection{Identify keys and design extended ER- diagram}

Abstract the functional dependencies amongst attributes of each entity from the business rules of the information system. Identify the primary key and foreign keys for each entity/actor. If an attribute or group of attributes of an entity of data store is independent of the primary key, take it out and form separate entity. The entities and attributes are abstracted from the data dictionary and the interrelationships are abstracted from the business rules of the system.

\subsection{Minimize the Imbalance between the Procedure and Data Oriented Paradigms}

[Fig-1.1 \&1.2] Regroup the attributes of input data flows, based on their characterization of Person, place, thing, event or concept. This can be achieved through the grouping the attributes such that each attribute of a group establishes a functional dependence with one primary key.

The input data flows may contain subset of attributes of a group so that each group of attributes may be in input data flows of one or more processes. Each such group forms a first cut object structure. [Fig-1.3] shows the perfect balance between data and procedure oriented i.e object oriented which

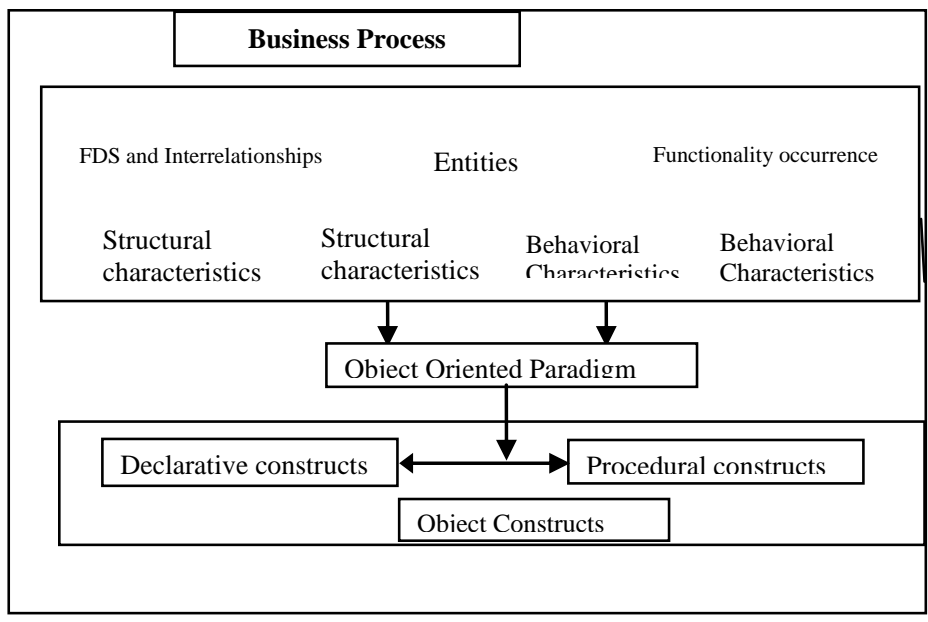

Figure-1.3 Object Oriented

\subsection{Refine the Abstracts by Brining the Perfect Balance between the Paradigms}

[5.6.7] Apply good database design principles to each first cut object structure [section 3.2].

Since the normalization is a way of good database design, the refined group should be normalized at least up to Boyce coded normal form. Figure-2.2 indicates the constructs to bring perfect balance between procedure oriented and data oriented paradigms.

\subsection{Design the Logical DFD with Object Structure}

Fig-2.2 Refine the logical data flow diagram with each object structure group as data store, maintaining attributes of total input data flow to each process and redesigning input flows such that each flow emanates from first cut object structure. 


\subsection{Decompose Functionality}

If two or more data flows directed towards single process, study the possibility of decomposing the process, so that each decomposed process either receives data flow from single entity or additionally through parameter passing from other entities. Refine the logical data flow diagram into physical data flow diagram.

\subsection{Software Requirement Specification [SRS]}

The input to the any information system is the requirements that contain the business rules of the information system along with various applications. For example, SRS for Banking Application information system may contain some of the business rules as follows.

\section{Context Level Diagram}

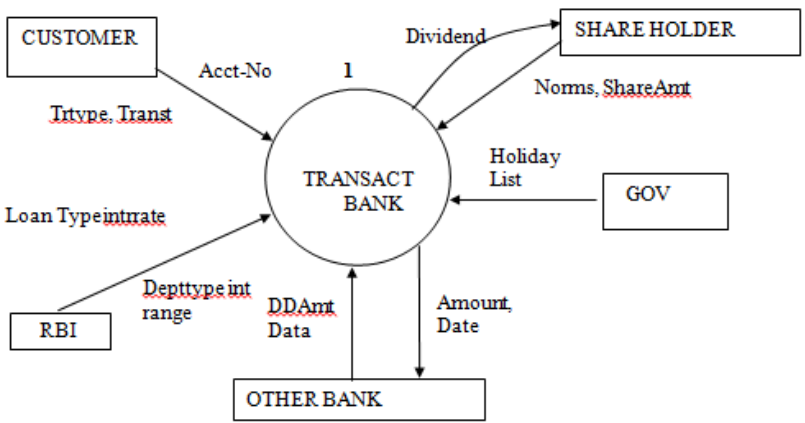

Figure-2.1 Context Level Diagram

Context Level Diagram [14]:

Fig-2.1 Depicts that the interaction between the banking information systems with the external agents which acts as data sources and data sinks.

\section{THEORY}

Automate the customer transaction in the banking system

There are two types of customer.

Deposit customer

- Each customer is provided with a unique account number

- Periodically credits/debits from his/her account.

- The customer earns interest on minimum balance of each month.

- There may be overdraft facility for some customer who has developed some kind of rapport with the bank.

- The customer can also transfer amount from his/her account to any account.

\section{Loan Customer}

- Loan customer initially applies for loan for specific purpose.

- The bank grants loan to customers after authenticating their repayments in installments.
- The bank charges interest either on floating-point rate or fixed-point rate.

- Interest rate for bank loan is generally much higher than interest rate for bank deposit

- Thus the bank earns profit by liaising between savings customer and loan customer

- Each branch manager needs to submit periodical summary report indicating the total number of transactions, the total amount deposited, the total interest paid to the customer, the total amount sanctioned, the interest earn by customer and thus the profit earn by the bank in the specific

\subsection{Identification of Classes attributes from SRS}

Depositor (Acctno,D-name,D-address,Contact-no)

Transaction (Acctno,Pre-balance,Transtype,Transamt,Newbalance, OD-facility)

Daccount (acctno, period, minibalance, int-rate, intAmt)

Transfer (acctno, minbalance,ch-amt,payeeacct,newbalance,payeebalance)

Borrower (acctno, loan-type, Amt-sanctioned, Amtavailed, newbalance, int-rate)

EMI (acctno, Balance, Rp-period, Principal, Int, EMI)

Baccount (acctno, period, maxbal, int-rate, intamt)

Report (period, no of depositor, amt deposited, int-paid, amtsanctioned, no of borrowers, amt-disbursed, int-earned, profit)

Loan (Bacctno, Bname, Baddress, Bcontact no)

\subsection{Synonyms and Homonyms}

These are Homonyms found in various entities like, Acctno, period, Int-rate, Int-amt, NewBalance.

Depositor (Acctno,D-name,D-address,Contact-no)

Transaction (Acctno,Pre-balance,Transtype,Transamt,Newbalance,OD-facility)

Daccount (Dacctno, Dperiod, minibalance, Dint-rate, DintAmt)

Transfer (Dacctno, minbalance,ch-amt,payeeacct,Dnewbalance,payeebalance)

Borrower (Bacctno, loan-type, Amt-sanctioned, Amtavailed, Bnewbalance, Bint-rate)

EMI (Bacctno, Balance, Rp-period, Principal, Int, EMI)

Baccount (Bacctno, Bperiod, maxbal, Bint-rate, Bintamt)

Report (period, no of depositor, amt deposited, int-paid, amtsanctioned, no of borrowers, amt-disbursed, int-earned, profit)

Loan (Bacctno, Bname, Baddress, Bcontact no)

I could not find any of synonyms in this application 


\section{DATA FLOW DIAGRAM (DFD) (Context Level Diagram and Logical DFD refer Page no.4, 5)}

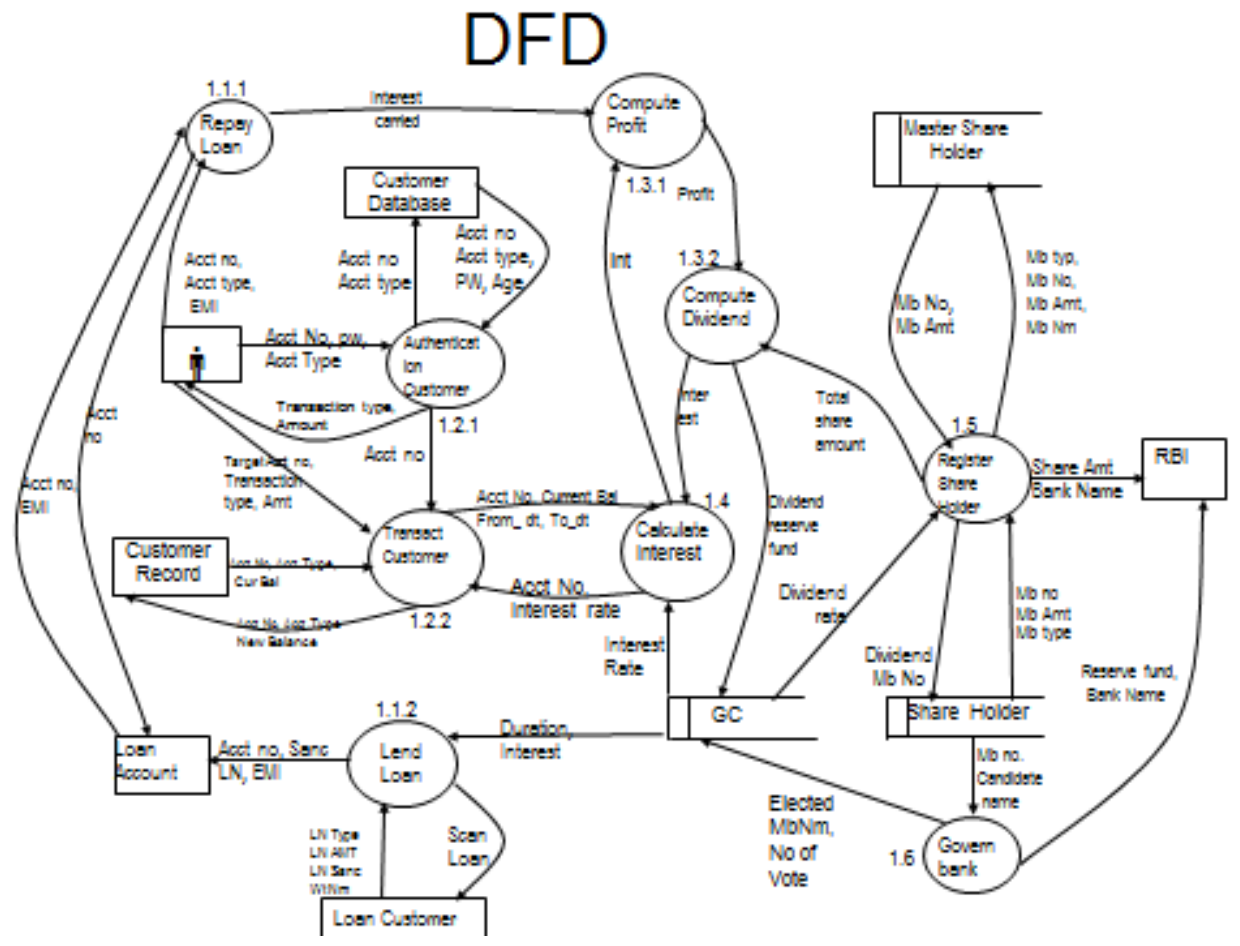

Figure-2.2 logical DFD [15] captures the data flows that are necessary for a banking information system to operate. It describes the processes that are undertaken, the data required and produced by each process

Results:

\section{Actors: Customer, Depositor}

Attributes of Actor: report, transaction, EMI, Bank database

Classes: Sanction Loan, Transaction Amount, Transfer Amount,Compute EMI,Prepare Report, Assign Account No.

Objects of Classes: Acctno,D-name,D-address,Contactno,Acctno,Pre-balance,Transtype,Transamt,New-balance,ODfacility) Dacctno, minbalance,ch-amt,payeeacct,Dnewbalance,payeebalance

\section{(Bacctno, Balance, Rp-period, Principal, Int, EMI)}

period, no of depositor, amt deposited, int-paid, amtsanctioned, no of borrowers, amt-disbursed, int-earned, profit, Bacctno, Bname, Baddress, Bcontact no.

\section{Discussion:}

Unified Modeling Language [UML][11,12] has become a de facto standard for the design and implementation of object oriented information system. Though the UML [13] is a boom for the design of business processes in an object oriented paradigm, the success rate of projects involving the design using UML is very slow.

The SRS is the abstraction of number of end user's requirements. Because of the environment in which the individual is working, the end user may use different words for the same meaning. Thus, the SRS contains synonymous words. In addition, the use of contexts specific words compels the end user to use the same word for different meanings. Thus, SRS also contains homonymous. The non-resolution of synonymous issue results in the redundancy model elements whereas; non-resolution homonym issue results in violation of uniqueness of the model elements. Thus any information system design needs to resolve the synonym and homonym issues. Thus, the success rate of the information system developed using such error prone approaches swings. There is an urgent need to isolate the dependency of software development success rate on human skills and to elevate it to the height of the automatic process.

\section{CONCLUSION}

UML is a graphical language for visualizing, specifying, constructing, and documenting the artifacts of a softwareinformation system. But suffer from the correctness and completeness aspect. Developed methodology helps in designing, design components (Classes, Objects and its Attributes) in a semiautomatic way incorporating correctness and completeness in each of its stages. This methodology minimizes human interventions that too giving clear-cut guidelines. In future these, intervention also can be automated.

\section{ACKNOWLEDGEMENT}

Firstly, I would like to express my sincere gratitude to my advisor Prof. Dr S.M.Handigund for the continuous support related research, for his patience, motivation, and immense knowledge. His guidance helped me in all the time.

\section{REFERENCES}

[1] Pankaj Jalote (2005). An Integrated approach to Software engineering, 3rd edition springer's publications, India.

[2] Roger S Pressman (2005). Software Engineering, 6th edition McGraw-Hill companies international edition, India 
[3] Mosa Emhamed Elbendak(2005) "Framework for using a Natural Language Approach to Object Identification Framework for using a Natural Language" http://www.aclweb.org/anthology/R09-2005.

[4] Muhammed Usman, Stepahane Ducane and Marianne Huchard (IEEE-2008) 15th working conference on reverse engineering "Reconsidering classes in procedural object oriented code" page 257- 266.

[5] Jonathan \& charles J Hannon (IEEE-2009) Eighth Mexican International Conference on Current Trends in Computer Science. "An algorithm for identifyingAuthors using synonyms" page $99-104$.

[6] Alen Lovrencie and Tonirnir Kisasondi (IEEE-2007) 11th International Conference on Intelligent Engineering System "Modelling Functional Dependencies in Databases using mathematical logic" page $307-312$.

[7] Steffen Rudle and LarsSchmidt-thieme (IEEE-2006) Sixth International Conference in Data Mining "Object Identification with constraints" page $1-7$.

[8] Sukhamay Kundu \& Migel (IEEE-2005) 29th International Conference Software and Application Conference "A Formal approach to Designing a class subclass structure using a partial order on the functions" page $1-8$.

[9] Mosa Emhamed Elbendak "Framework for using a Natural Language Approach to Object Identification Framework for using a Natural Language" Student
Research Workshop, RANLP 2009 - Borovets, Bulgaria, pages $23-28$

[10] .S. Anandha Mala and Dr.G.V.Uma "Object Oriented Visualization of Natural Language Requirement Specification and NFR Preference Elicitation. IJCSNS International Journal of Computer Science and Network Security, VOL.6 No.8, August 2006.

[11] Lilac A. E. Al-Safadi "Natural Language Processing for Conceptual Modeling" A semi-automatic approach for designing databases. International Journal of Digital Content Technology and its Applications Volume 3, Number 3, September 2009

[12] Sunguk Lee "UML for Database System and computer application. Interantional Journal of Database System and Computer Application. Volume 5, number 3, 2012

[13] K.P Jayant, Garg,Kumar, Rana. "An approach of Software Design Testing Based on UML diagram. International Journal of Advanced Research in Computer Science and Software Engineering. Volume-4, Issue-2, February 2014

[14] Tiwari,Alpika,Dubey "Merging of Dataflow Diagrams with Unified Modeling Language" Internation Journal of Scientific and Research Publication Volume-2,issue-8, August-2012

[15] Rosziati,Yen "A Formaol Model for Data Flow Diagrams Rules" APRN Jounal of System and Software, volume-1 No.2, May-2011 